\title{
Multirate-aware Multicast Routing in MANETs
}

\author{
Uyen Trang Nguyen, Amir Asif, and Xing Xiong \\ Department of Computer Science and Engineering \\ York University, Toronto, Canada M3J 1P3 \\ Email: \{utn, asif, xing $\} @$ cs.yorku.ca
}

\begin{abstract}
We propose a Rate-Adaptive Multicast (RAM) routing protocol for mobile ad-hoc networks (MANETs) that is multirate-aware. During the process of path discovery, the quality of wireless links is estimated to suggest optimal transmission rates, which are then used to calculate the total transmission time incurred by the mobile nodes on a path. Among several considered paths from a source to a destination, RAM selects the path with the lowest total transmission time. Simulation results have shown that RAM outperforms single-rate multicast in terms of packet delivery ratio, packet end-to-end delay, and throughput of the multicast group. In this paper, we apply RAM to effectively support video multicast in MANETs.
\end{abstract}

\section{INTRODUCTION}

A current trend in wireless communications is to enable wireless devices to transmit at different rates at the physical layer. Many standards supporting this capability have been made available, such as 802.11a, 802.11b, 802.11g, and HiperLAN2. For example, $802.11 \mathrm{~b}$ specifies rates of $1 \mathrm{Mbps}$, $2 \mathrm{Mbps}, 5.5 \mathrm{Mbps}$ and $11 \mathrm{Mbps}$. Rate adaptation is the process of dynamically switching data rates to match the channel conditions in order to obtain the optimal throughput.

Multicast refers to one-to-many or many-to-many communications. Important multicast applications include audio/video conferencing, distance education, interactive games, and distribution of financial data, software and newspapers. Wireless adhoc networks are well suited for multicast due to their inherent broadcast capability. Several multicast routing protocols have been proposed specifically for MANETs [1], [2].

Currently most multicast routing protocols tend to select the shortest path from a sender to a receiver. Such a path contains a minimum number of hops between the source and the destination. Therefore, the distances between hops are long, and the transmission rates are kept low. A low transmission rate incurs long transmission time, which results in low throughput and high energy consumption.

We expect that another route selection criterion, combined with multi-rate transmission, can improve the situation. For example, we could choose paths that are slightly longer than the shortest paths (i.e., having more hops) but on which the distances between neighboring nodes are shorter. Shorter distances between multicast nodes permit data transmissions at higher rates, thereby, lowering transmission time, increasing network throughput and reducing the energy consumption.

Specifically, we propose a Rate-Adaptive Multicast (RAM) routing protocol based on the on-demand multicast routing protocol (ODMRP) [2]. RAM is a multirate-aware routing protocol, which works as follows. During the process of path discovery, the quality of wireless links is estimated to suggest optimal transmission rates, which are then used to calculate the total transmission time incurred by the mobile nodes on a path. Among several considered paths from a source to a destination, the routing protocol selects the path with the lowest total transmission time. Our previous experimental results show that RAM outperforms single-rate multicast in almost all scenarios, in terms of packet delivery ratio, packet end-to-end delay, and throughput of the multicast group [3]. In this paper, we apply RAM to implement a framework for video multicast in MANETs.

The remainder of the paper is organized as follows. We discuss related work in Section II. The proposed RAM protocol is described in Section III. In Section IV, we present the design, implementation and performance evaluation of the proposed video multicast framework. Section V concludes the paper.

\section{RELATED WORK}

Rate adaption involves two stages: channel quality estimation and rate selection. Several metrics are used as indicators of channel quality such as signal-to-noise ratio, signal strength, symbol error rate, or bit error rate. The rate selection procedure then uses the channel quality estimate to select an appropriate rate. A common technique of rate selection is to compare the value of the channel quality indicator against a list of threshold values representing boundaries between the data rates [4].

To estimate the channel quality, the Autorate Fallback (ARF) rate adaptation scheme, implemented in Lucent's 802.11 WaveLAN-II networking devices, uses the receipts or absence of acknowledgments (ACKs) as an indication of channel quality [5]. The Receiver-Based Autorate (RBAR) protocol [6], on the other hand, uses the strength of received signals. The latter method provides faster and more accurate estimate than the use of ACKs. The protocol proposed by Pavon and Choi for WLANs [7] combines the use of signal strength and the frame error rate to determine the transmission rate. All of the aforementioned protocols were originally designed for WLANs and can also be applied to MANETs [8]. However, these rate-adaptation protocols operate independently of the routing protocol. Therefore, if the routing protocol finds a short path with long hops, the rate-adaptation protocol may not be able to use high rates. The RAM protocol we propose incorporates rate selections into the routing protocol for optimal performance.

The Opportunistic Media Access (OMA) protocol [9] uses RBAR for channel quality estimation and rate selection. Once 
the rate is calculated and the channel is reserved, the source will send for a duration as if the base rate were used. That is, a flow with a data rate of $11 \mathrm{Mbps}$ will transmit 5.5 times as many packets as a flow with a data rate of $2 \mathrm{Mbps}$. This scheme aims at achieving temporal fairness, as opposed to throughput fairness in RBAR.

The rate-adaptation schemes proposed by Qiao et al. for WLANs [10] and Awerbuch et al. for MANETS [11] are based on theoretical models of the attainable throughput in WLANs/MANETS. The models permit us to compute link rates that maximize the throughput. The models, however, make simplifying assumptions (e.g., all nodes in the network are within each other's transmission range [11]), and the rate computation assumes that the packet size is known in advance and that all packets have the same size.

A multirate-aware routing protocol for MANETs was proposed by Seok et al. [12]. The protocol requires a new sublayer called Multirate Aware Sub-layer (MAS) to be added between the network layer and the MAC layer, which requires considerable modifications to the standards.

The RAM protocol we propose is the first multirate-aware routing protocol for multicast. It requires no modifications to the standards and offers significant improvement over singlerate multicast as proved by our simulation results [3].

\section{THE RAM PROTOCOL}

In unicast communications, the transmission of a data packet is preceded by an RTS/CTS (Request To Send/Clear To Send) exchange. The receiving node estimates the channel quality based on the signal strength of the received RTS and sends the rate information to the sending node via the CTS. Since there is no RTS/CTS exchange in multicast, we propose to use the control packets of the routing protocol for estimating the channel quality. Specifically for ODMRP, channel conditions are estimated based on the signal strength of the received JOIN_QUERY packets. The receiving node then sends the rate information to the sending node using the JOIN_REPLY packets. The rate information is thus updated with each route refreshment. This scheme allows the control packets to be used in an efficient manner, for both routing and rate adaptation.

JOIN_QUERY packets are used for estimating the channel conditions, and for accumulating the weights of the links on the paths they traverse in a field called pathWeight. Given the optimal transmission rate $R$ of a link (as determined by the signal strength of a received packet), the weight of the link is defined as $1 / R$. The weight of a path is the sum of the weights of all the links on that path. After receiving a JOIN_QUERY, every node on a path between a sender and a receiver measures the signal strength of the packet, which is then compared against a set of threshold values to suggest a transmission rate, SuggestedRate, using an algorithm similar to RBAR [8]. The SuggestedRate is converted to the link weight, which is then added to the pathWeight recorded in the JOIN_QUERY. When the JOIN_QUERY arrives at a receiver, field pathWeight contains the weight of the sourceto-destination path traversed by the JOIN_QUERY.
A node participating in the route discovery/refresh process may receive multiple JOIN_QUERY packets from the same sender that take different paths. The node examines all these JOIN_QUERY packets and selects the JOIN_QUERY, and hence the path, with the minimum pathWeight value. This process is termed as the JOIN_QUERY consolidation step.

After a receiver performs the JOIN_QUERY consolidation, the selected path is the source-to-destination path incurring the lowest total transmission time among those examined. The receiver then creates a JOIN_REPLY that records the routing information of this path, and sends the JOIN_REPLY to the source, as in the ODMRP algorithm. To prevent the implosion of JOIN_REPLY packets, every node on the paths from the receivers to the sender consolidates information from several JOIN_REPLY packets it receives from downstream ${ }^{1}$ nodes into one JOIN_REPLY, as in ODMRP. This process is termed as the JOIN_REPLY consolidation step.

Note that all transmissions of JOIN_QUERY and JOIN_REPLY packets use the base rate (e.g., 1 Mbps in 802.11b) to ensure that they are received correctly over long links. Data packets are transmitted at the optimal rates selected based on channel conditions. A detailed description of the RAM protocol can be found in [3]. Previous simulation results [3] have shown that RAM offers much better performance than single-rate multicast with respect to packet delivery ratio, throughput and packet end-to-end delay.

\section{A Framework For Video Multicast in MANETS}

Video multicast over MANETs is a challenging problem due to heterogeneities and constraints such as low-bandwidth wireless channels and limited battery power. Video applications, on the other hand, are demanding in terms of bandwidth and delay constraints. We propose a framework for multicasting video over MANETs, which employs the RAM protocol instead of single-rate multicast to meet the requirements of video applications.

\section{A. Framework Design}

We solve the heterogeneity and constraint issues of video multicast over MANETs as follows.

- Limited bandwidth. The video multicast framework uses the scalable, noncausal predictive codec with vector quantization and conditional replenishment (SNP/VQR) [13], which offers superior video quality than the standard codecs including MPEG4 and H.264 at low bit rates. Use of SNP/VQR allows transmission at a lower rate than required by the standard codecs with no perceivable difference in the video quality. To further overcome the bandwidth limitation, we employ the RAM protocol instead of single-rate multicast to achieve higher packet delivery ratio and higher throughput.

- Network/receiver heterogeneities. In order to accommodate a wide range of mobile devices, SNP/VQR offers layer coding where the video is encoded into multiple layers of data streams. A subset of the compressed bit stream provides the

\footnotetext{
${ }^{1}$ The downstream direction is from a sender towards a receiver. Conversely, the upstream direction is from a receiver to a sender.
} 
base quality. Additional enhancement layers build on to the video quality in both the spatial and temporal domains. Receivers with powerful batteries and access to higher bandwidth channels can choose to receive all layers, thus acquiring video of the highest quality. Other receivers may opt for a reduced number of layers to save energy and network bandwidth. Similarly, when a router detects the onset of congestion or a drained battery, it can select to transmit only the most important layer(s) using priority packet dropping [14].

- Limited battery power. This problem is solved in part by using layered video coding and priority packet dropping as mentioned above. In addition, our RAM protocol allows routers to save energy by minimizing the transmission time.

\section{B. Framework Implementation}

A SNP/VQR encoder [13] at the sender compresses the video sequence frame by frame. The sender then transforms the compressed frames into an array of data packets, which are transmitted over the MANET. In our framework, we implement three levels of quality of service: gold, silver and bronze. The bronze service uses only the base (the most important) layer. The silver service couples the base and first enhancement layers, while the gold service requires all three layers. A receiver can decide how many layers it wants to receive. After the selected layers are received, the SNP/VQR decoder reconstructs the compressed video frames. In case of transmission errors, the SNP/VQR decoder applies error concealment techniques [15] to the damaged pixels.

To multicast layer coded video over a network, either of the following approaches can be used: priority packet dropping (PPD) [14], and receiver-driven layered multicast (RLM) [16]. We found that RLM is not suitable for layered multicast in MANETs due to the following reasons. First, the join experiments adds adversely to the network traffic, especially when the size of the multicast group is large. Second, RLM does not perform well in tracking available bandwidth in a bursty network [14]. Furthermore, RLM is much more complex to implement than PPD. We thus chose PPD for multicasting layered video in our framework.

We also implemented the RAM protocol in the framework, which provides the routing function of ODMRP and rate adaptation. In the next section, we present experimental results that show the effectiveness of the proposed framework.

\section{Performance Evaluation}

We evaluated the performance of our video multicast framework using several video sequences under different network conditions. The network component is simulated using QualNet [17], a commercial software that provides scalable simulations of wireless networks. We present here the results from one set of experiments in which a video sequence with 120 frames was encoded and transmitted over the simulated MANET. The simulated network consisted of 50 mobile nodes randomly placed within a $1000 \mathrm{~m} \times 1000 \mathrm{~m}$ area with each mobile host moving at a speed of $1 \mathrm{~m} / \mathrm{s}$. One node was randomly selected as the sender of a multicast session
TABLE I

SIMULATION PARAMETERS

\begin{tabular}{|l|l|}
\hline Parameter & Value \\
\hline ODMRP route refresh interval & 20 seconds \\
ODMRP forwarding timeout & 60 seconds \\
Mobility model & Random way-point [18] \\
Pathloss model & Two-ray [19] \\
Node transmission power & $15 \mathrm{dBm}$ \\
Physical layer protocol & PHY802.11b \\
Medium access control & MAC802.11. Multicast uses CSMA/CA \\
& Unicast uses CSMA/CA and RTS/CTS \\
Queue size at each router & 50Kbytes \\
Queuing policy at routers & PPD - FIFO within each queue \\
\hline
\end{tabular}

TABLE II

DISTRIBUTION OF VIDEO PACKETS LOST

\begin{tabular}{|l|c|c|c|c|c|}
\hline \multirow{2}{*}{ Layer } & \multirow{2}{*}{ Packets } & \multicolumn{2}{|c|}{$\begin{array}{r}\text { Packets Lost in } \\
\text { Light } \text { Traffic }\end{array}$} & \multicolumn{2}{c|}{$\begin{array}{c}\text { Packets Lost in } \\
\text { Heavy Traffic }\end{array}$} \\
\cline { 3 - 6 } & Number & Rate & Number & Rate \\
\hline \hline Bronze & 4,880 & 70 & $1.43 \%$ & 196 & $4.02 \%$ \\
\hline Silver & 4,880 & 99 & $2.03 \%$ & 320 & $6.56 \%$ \\
\hline Gold & 4,800 & 175 & $3.65 \%$ & 3,276 & $68.25 \%$ \\
\hline Total & 14,400 & 344 & $2.70 \%$ & 3,795 & $26.28 \%$ \\
\hline
\end{tabular}

having 20 receivers. The receivers were selected with uniform probability among the 50 mobile nodes. The members joined the multicast group at the beginning of the simulation and stayed on until the whole group was terminated. The output from the SNP/VQR codec was compressed video packets marked as "gold", "silver" and "bronze" packets. The sender transmitted the packets over three respective multicast groups, each corresponding to a layer. The sending rate for each group is 60 packets/s, which corresponds to the output rate of the $\mathrm{SNP} / \mathrm{VQR}$ encoder. The packet size excluding the header size varied from 99 to 250 bytes. Other network parameters are listed in Table I.

We measure the packet loss rates for the three video layers under both light and heavy traffic conditions. The results are shown in Table II, where the packet loss rates averaged over the 20 receivers are given. We can see that the majority of lost packets in the heavy load network are from the gold layer (the least important layer) due to priority packet dropping.

The quality of reconstructed video frames is assessed by computing the average peak signal-to-noise ratio (PSNR), defined as

$$
\text { Average PSNR }=\frac{1}{R \times N_{K}} \sum_{r=1}^{R} \sum_{k=1}^{N_{K}} \operatorname{PSNR}(k, r),
$$

where $\operatorname{PSNR}(k, r)$ is defined as the PSNR of frame $k$ reconstructed by receiver $r, N_{K}$ is the total number of frames in the video sequence, and $R$ is the number of receivers included in the multicast session. Table III lists the average PSNRs under three different network conditions: no packet loss, low packet loss under light traffic, and moderate packet loss under heavy traffic. We observe that the quality of the reconstructed video in the light load network is comparable to that in an ideal setting with no packet loss. The video quality remains reasonably good even in the network with high traffic. 

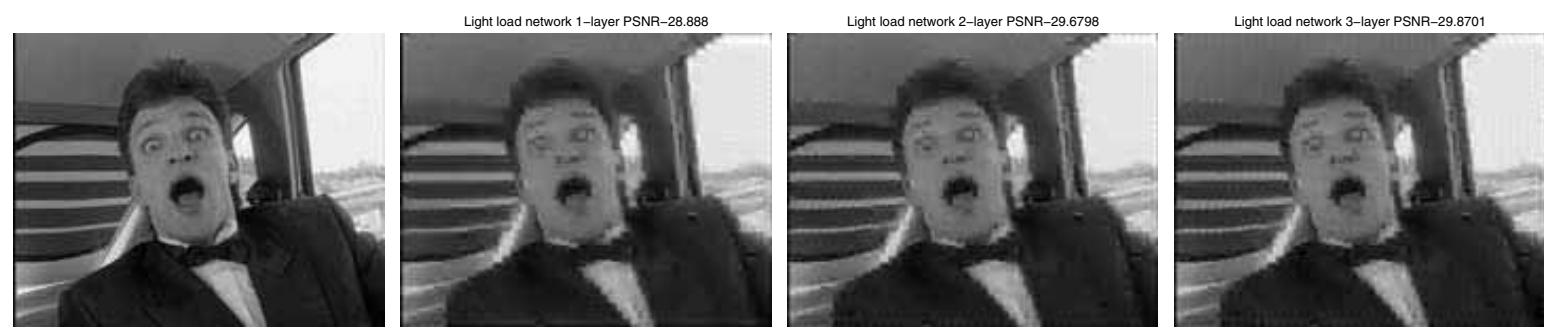

(a) Original frame

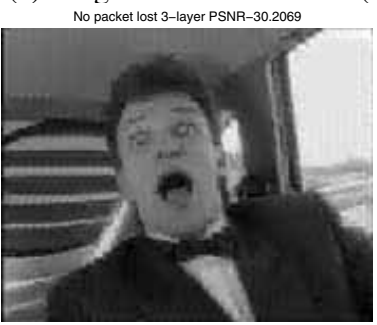

(b) Light traffic, bronze service Heavy load network 1-layer PSNR-28.686
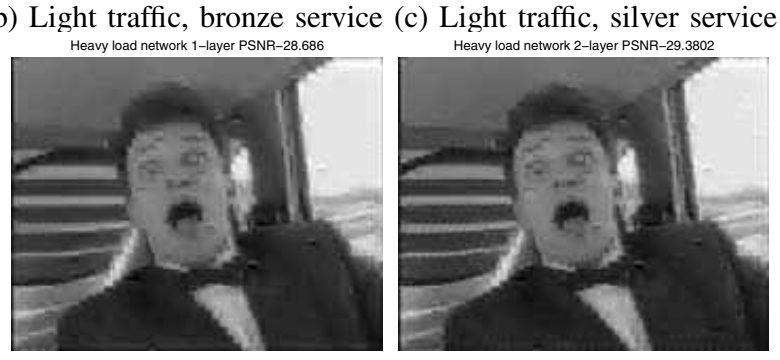

(d) Light traffic, gold service

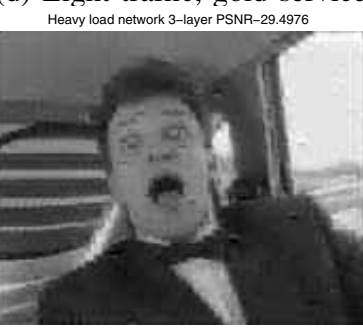

(e) No loss, gold service (f) Heavy traffic, bronze service (g) Heavy traffic, silver service (h) Heavy traffic, gold service

Fig. 1. Perceived video quality from bronze, silver and gold services

TABLE III

AVERAGE PSNRS FOR BRONZE, SILVER, AND GOLD SERVICES

\begin{tabular}{|l|c|c|c|c|}
\hline Network & Packets & \multicolumn{3}{|c|}{ Quality of Service } \\
\cline { 3 - 5 } Conditions & Lost & Bronze & Silver & Gold \\
\hline \hline Ideal (no loss) & $0 \%$ & $28.75 \mathrm{~dB}$ & $29.58 \mathrm{~dB}$ & $29.99 \mathrm{~dB}$ \\
\hline Light traffic & $2.70 \%$ & $28.59 \mathrm{~dB}$ & $29.32 \mathrm{~dB}$ & $29.69 \mathrm{~dB}$ \\
\hline Heavy traffic & $26.28 \%$ & $28.36 \mathrm{~dB}$ & $28.88 \mathrm{~dB}$ & $29.00 \mathrm{~dB}$ \\
\hline
\end{tabular}

To provide a subjective evaluation of the quality of the reconstructed video, Figure 1 includes several frames reconstructed by the SNP/VQR decoder at different receivers. Since the quality of the reconstructed video varies from one receiver to another, we chose frames from video sequences whose average PSNR is closest to the PSNR values included in Table III. The sample frames demonstrate that our framework offers very good perceptual visual quality, even in congested networks. This is due in large part to the high performance provided by the proposed RAM protocol.

\section{CONCLUSION}

We propose a multirate-aware routing protocol for multicast in MANETs. Channel conditions are estimated, and the resulting optimal transmission rates are communicated using the control packets of the routing protocol. Among several paths available between a sender and a receiver, the routing protocol selects the path with the lowest total transmission time. Simulation results have shown that the proposed RAM protocol outperforms single-rate multicast in terms of packet delivery ratio, end-to-end delay, and throughput. RAM's high performance enables effective video multicast in MANETs, as demonstrated by our proposed video multicast framework.

\section{REFERENCES}

[1] C. de Morais Cordeiro, H. Gossain and D. P. Agrawal, "Multicast over wireless mobile ad hoc networks: Present and future directions," IEEE Network, Jan. 2003.
[2] S. J. Lee, W. Su, and M. Gerla, "On-Demand Multicast Routing Protocol in Multihop Wireless Mobile Networks," ACM/Kluwer Mobile Networks and Applications, Dec. 2002.

[3] U.T. Nguyen and X. Xiong, "Rate-adaptive Multicast in Mobile Adhoc Networks," IEEE Int. Conf. on Wireless and Mobile Computing, Networking and Communications 2005, Aug. 2005.

[4] K. Balachandran, S. Kadaba, and S. Nanda, "Channel quality estimation and rate adaption for cellular mobile radio," IEEE JSAC, Jul. 1999.

[5] A. Kamerman and L. Monteban, "WaveLAN-II: A high-performance wireless LAN for the unlicensed band," Bell Labs Technical Journal, vol. 2, 1997.

[6] G. Holland, N. Vaidya, and P. Bahl, "A rate-adaptive MAC protocol for multi-hop wireless networks," Technical Report TR00-019, Dept. of Computer Science, Texas A \& M University, Aug. 2000.

[7] J. Pavon, and S. Choi, "Link adaptation strategy for IEEE 802.11 WLAN via received signal strength measurement," Proc. of IEEE ICC, May 2003.

[8] G. Holland and N. H. Vaidya and P. Bahl, "A rate-adaptive MAC protocol for multi-hop wireless networks," Proc. of ACM MOBICOM, Jul. 2001.

[9] B. Sadeghi, V. Kanodia, A. Sabharwal, E. Knightly, "Opportunistic media access for multirate ad hoc networks," Proc. of ACM MOBICOM, 2002.

[10] D. Qiao, S. Choi, and K. Shin, "Goodput analysis and link adaptation for IEEE 802.11a wireless LANs," IEEE Trans. on Mobile Computing, Sept. 2002.

[11] B. Awerbuch, D. Holmer, and H. RubensHigh, "Throughput route selection in multi-rate ad hoc wireless networks," Technical Report, Johns Hopkins University, Mar. 2003.

[12] Y. Seok J. Park, and Y. Choi, "Multi-rate aware routing protocol for mobile ad hoc networks," Proc. of IEEE Vehicular Technology Conference, Spring 2003, Apr. 2003.

[13] A. Asif and M. G. Kouras, "Scalable Video Codec by Noncausal Prediction, Cascaded Vector Quantization, and Conditional Replenishment," IEEE Transactions on Multimedia, vol. 8, no. 1, Feb. 2006.

[14] S. Bajaj, L. Breslau, and S. Shenker, "Uniform versus Priority Dropping for Layered Video," ACM SIGCOMM Comp. Comm. Review, 1998.

[15] Y. Wang and Q.-F. Zhu, "Error control and concealment for video communication: A Review," Proceedings of the IEEE, vol. 86, no. 5, May 1998.

[16] S. McCanne, V. Jacobson, and M. Vetterli, "Receiver-driven Layered Multicast," ACM SIGCOMM, Aug. 1996.

[17] QualNet Network Simulator, www.scalable-networks.com.

[18] J. Yoon, M. Liu, and B. Noble, "Random waypoint considered harmful," Proc. of IEEE INFOCOM, Apr. 2003.

[19] T.S. Rappaport and L.B. Milstein, Effects of Radio Propagation Path Loss on DS-CDMA Cellular Frequency Reuse Efficiency for the Reverse Channel, IEEE Transactions on Vehicular Technology, Vol. 41, No. 3, Aug. 1992 